\title{
Barriers to Formal Emergency Obstetric Care Services' Utilization
}

\author{
Hildah Essendi, Samuel Mills, and Jean-Christophe Fotso
}

\begin{abstract}
Access to appropriate health care including skilled birth attendance at delivery and timely referrals to emergency obstetric care services can greatly reduce maternal deaths and disabilities, yet women in sub-Saharan Africa continue to face limited access to skilled delivery services. This study relies on qualitative data collected from residents of two slums in Nairobi, Kenya in 2006 to investigate views surrounding barriers to the uptake of formal obstetric services. Data indicate that slum dwellers prefer formal to informal obstetric services. However, their efforts to utilize formal emergency obstetric care services are constrained by various factors including ineffective health decision making at the family level, inadequate transport facilities to formal care facilities and insecurity at night, high cost of health services, and inhospitable formal service providers and poorly equipped health facilities in the slums. As a result, a majority of slum dwellers opt for delivery services offered by traditional birth attendants (TBAs) who lack essential skills and equipment, thereby increasing the risk of death and disability. Based on these findings, we maintain that urban poor women face barriers to access of formal obstetric services at family, community, and health facility levels, and efforts to reduce maternal morbidity and mortality among the urban poor must tackle the barriers, which operate at these different levels to hinder women's access to formal obstetric care services. We recommend continuous community education on symptoms of complications related to pregnancy and timely referral. A focus on training of health personnel on "public relations" could also restore confidence in the health-care system with this populace. Further, we recommend improving the health facilities in the slums, improving the services provided by TBAs through capacity building as well as involving TBAs in referral processes to make access to services timely. Measures can also be put in place to enhance security in the slums at night.
\end{abstract}

KEYWORDS Urban poor, Nairobi, Slums, Formal Emergency Obstetric Care (EmOC), Barriers, Qualitative data

\section{BACKGROUND}

Evidence indicates that access to appropriate health care including skilled birth attendance at delivery and timely referrals to emergency obstetric care services can greatly reduce maternal deaths and disabilities ${ }^{1-4}$. In most countries in sub-Saharan Africa, however,

\footnotetext{
Essendi is with the Centre for Global Health, Population, Poverty and Policy (GHP3). Faculty of Social and Human Sciences, University of Southampton, Southampton, United Kingdom; Mills is with the World Bank, Washington, DC, USA; Fotso is with the African Population and Health Research Centre (APHRC), Nairobi, Kenya.

Correspondence: Hildah Essendi, Centre for Global Health, Population, Poverty and Policy (GHP3). Faculty of Social and Human Sciences, University of Southampton, Southampton, United Kingdom. (E-mail: H.Essendi@soton.ac.uk)
} 
women continue to have very limited access to skilled delivery services ${ }^{3,5}$. The high prevalence of maternal illnesses, near misses, and other potentially devastating and acute obstetric complications suffered by women in the region have generally been linked to their poor access to emergency obstetric services ${ }^{1,6}$. While almost $100 \%$ of births in developed regions occur with skilled birth attendants, more than half of all births in subSaharan Africa still take place without the assistance of skilled birth attendants 5,7 . The situation is worse in the Eastern Africa region (where Kenya lies) with only 34\% of births assisted by skilled birth attendants compared with $41 \%$ in Western Africa. Consequently, of the estimated total of 536,000 maternal deaths worldwide in 2000, developing countries accounted for $99 \%(533,000)$, and slightly more than half of these $(270,000)$ occurred in the sub-Saharan Africa region alone ${ }^{8}$.

Kenya, like many other sub-Saharan African countries, experiences high maternal mortality and low skilled attendance at birth ${ }^{9}$. The 2003 Kenya Demographic and Health Survey estimates that only about $42 \%$ of women in the country deliver with the assistance of a health professional with $26 \%$ delivering in public health facilities. The maternal mortality ratio in the country is 414 deaths per 100,000 live births. These indicators are worse among the urban poor. A recent study in the Korogocho and Viwandani slums in Nairobi indicates maternal mortality ratio to be at 706 deaths per 100,000 live births ${ }^{10}$. It has been documented that the urban poor face worse health indicators than their rural counterparts or their counterparts in the urban non-poor settlements, challenging the commonly held assumption that the urban populace enjoys easy access to health services ${ }^{11}$. In the past few decades, the country has faced deteriorating economic growth and increasing population, conditions which have facilitated the growth of slums areas. In Nairobi, then majority of city residents live in these slums areas ${ }^{12}$. Life in these slum areas is associated with poor health indicators due to inadequate access to clean water, electricity and health facilities, and generally poor sanitation ${ }^{11,13,14}$. Only $52 \%$ of deliveries inNairobi slums occurred with skilled birth attendants, as compared with $78 \%$ citywide 9 . Although poverty, coupled with high costs of health services, poor transportation, and inadequate and inappropriately equipped health facilities, has been documented to be a serious barrier in the utilization of maternal health services among the rural poor ${ }^{15,16}$, little is known about the dimensions of these barriers among the urban poor. The areas of study-the Korogocho and Viwandani slums of Nairobi-have inadequate and poorly equipped health facilities just like many other urban slums. They are mainly served with privately owned, substandard, unlicensed, and informal health facilities ${ }^{17}$. Further, the exact effects of the identified barriers to the uptake of formal emergency obstetric services by this populace are not known.

While seeking to understand the barriers to health care services by sub-Saharan African women, many studies have focused on individual, household, community, and health system-level barriers ${ }^{18-21}$. From these studies, a lot is known about the demand and supply of these services. The effect of individual and household factors has also been documented ${ }^{22}$. The bulk of data used in these studies has, however, been collected using quantitative measures that ignore the direct views and accounts of the people who themselves use these services. Where it exists, qualitative research on the barriers to the use of formal delivery services have focused on rural women and men $^{19}$. Driven largely by the assumption that most women who are unable to utilize formal maternal health services reside in rural areas, research has ignored the experiences of millions of city women in Africa who also face poor access to quality birthing services. Yet, in advancing knowledge on the barriers to formal emergency obstetric care services, the experiences and views of urban poor women and their households are very critical. In Kenya, research shows that despite near universal 
utilization of antenatal care, a large number of women in urban areas continue to deliver at home like their rural counterparts ${ }^{9}$. This is an irony of the urban context where health facilities are supposed to be within reach, as compared with rural areas. In fact, a majority of the urban poor lack stable and regular sources of income, and the high cost of living in cities results in residents-and women in particularengaging in ad hoc jobs and trading during business hours when most health facilities are in operation ${ }^{23}$.

The purpose of this study is to investigate poor urban Kenyan men's and women's views on the factors that hinder the uptake of formal obstetric care services. Understanding lay perspectives regarding obstetric care services is paramount in developing programs and policies that will respond effectively to the sensitivities and needs of their expected beneficiaries. The use of qualitative methods in this study will help to understand these barriers at the individual, community, and health facility levels from the perspective of slum dwellers. Unlike population-based surveys, which are mostly conducted with structured questionnaires, the focus group discussions employed in this study allowed the barriers faced in the utilization of obstetric services to be explored in depth.

\section{METHODS}

\section{Study Area and Population}

The study settings are Viwandani and Korogocho, two informal settlements located in Nairobi, the capital city of Kenya. In these two settlements the African Population and Health Research Centre (APHRC) operates the Nairobi Urban Health and Demographic Surveillance System (NUHDSS) with about 60,000 registered inhabitants. The NUHDSS has monitored vital events like births, deaths, migration, and livelihood events of inhabitants of these two slum communities since 2001. Viwandani is located about $7 \mathrm{~km}$ southeast from Nairobi's city center and is bordered by the city's industrial area and the Nairobi River. It covers $0.52 \mathrm{~km}^{2}$ with a population density of 52,583 inhabitants $/ \mathrm{km}^{2}$. Korogocho covers a smaller area than Viwandani $\left(0.45 \mathrm{~km}^{2}\right)$ and has higher population density $(63,318$ inhabitants/ $\mathrm{km}^{2}$ ). It is located about $12 \mathrm{~km}$ east of the city center and is on reserve land of the City Council. Compared with Viwandani, Korogocho has less population disparity with regard to sex and age distribution.

\section{Data Collection}

This study uses qualitative data from a maternal health project implemented in 2006 in the Korogocho and Viwandani slums. The purpose of the project, which is part of a multi-country study involving the Kassena-Nankana District in northern Ghana and the state of Uttar Pradesh in India, was to provide a better understanding of the delays and barriers to emergency obstetric care utilization in low-resource urban settings in Nairobi. This project was conducted within the NUHDSS, and data were collected through household interviews and a health facility survey. From the NUHDSS database, all women aged between 12 and 54 years who had a pregnancy outcome in 2004-2005 were selected and interviewed. From this group, those who had life-threatening obstetric complications and failed to seek health care were purposively sampled and participated in focus group discussions. The complications reported were similar across the slums and age groups. They included abdominal pain, headache and swelling of the feet, high fever, blurry vision, prolonged labor, 
and excessive vaginal bleeding. Their partners, opinion leaders, traditional birth attendants (TBAs), and older women were also purposively sampled and participated in focus group discussions (see Table 1). In total, 16 focus group discussions (FGDs) were held with each of the groups, formed along similar socio-economic and demographic characteristics. Groups of women who had complications were composed based on demographic and slum residence status. Opinion leaders were selected in consultation with the chiefs. Ethical approval of the study was obtained from the Kenya Medical Research Institute's Ethical Committee and informed consent was obtained from participants before the discussions were held.

Six trained field workers ( 3 male and 3 female) conducted the interviews in Kiswahili." The FGDs were conducted using an FGD interview guide. All the interviews were audio recorded and transcribed into English. The areas of investigation in this study included the respondents' perceptions of formal delivery care services and the barriers that the community experiences to utilizing formal obstetric care services.

\section{Data Processing and Analysis}

Transcribed Word files were imported into NUD*ST 6 software (QSR International Pty Ltd, Australia) for coding ${ }^{24}$. This involved the continual reading of the transcripts and investigation of the themes emerging from the data for categories, linkages, and properties. In many instances, verbatim quotations were used to illustrate responses on relevant issues and themes.

\section{RESULTS}

\section{Perception of Formal ${ }^{\dagger}$ Delivery Services}

Responding men and women stated a preference for formal delivery services. They said that skilled birth attendants have the capacity to professionally attend to birthing mothers even in emergency cases. These personnel are considered trained to handle deliveries and any pregnancy-related complications. They are also able to correctly prescribe treatment and are preferred over informal providers. Health facility delivery was preferred because of the availability of the necessary medicines and equipment and the high level of hygiene. Discussants said that they could therefore entrust the lives of the mother and baby in the care of health personnel. They reported that seeking delivery services in formal health facilities could lessen delays in referrals to better facilities/health professionals as the

\footnotetext{
"Kiswahili is the commonly spoken language in the informal settlements of Nairobi .

$\dagger$ From the health facility survey, health facilities were classified into two categories. The first category, herein referred to as "appropriate," comprises health facilities run/owned by government, large NGOs, religious, and missionary groups that provide at least the basic essential obstetric care. These facilities are larger and are located in the outskirts of the slums; some are hospitals-two of which serve as referral facilities. They are able to offer most of the signal functions of basic emergency obstetric care, as defined by the following six procedures: administration of parenteral antibiotics, administration of parenteral oxytocic drugs, administration of parenteral anticonvulsants (for pre-eclampsia), manual removal of retained products of conception, manual removal of a retained placenta, and assisted vaginal delivery (vacuum extraction). These are formal health facilities. The second category, herein referred to as "inappropriate," comprises the substandard health clinics that do not provide basic essential obstetric care services. These are mainly privately owned and unlicensed clinics located within the two slum communities. They are informal facilities.
} 
TABLE 1 Characteristics of groups purposively sampled for focus group discussions

\begin{tabular}{lll}
\hline Group & Age category (years) & No. of groups \\
\hline Women & $12-19$ & 2 \\
Women & $20-29$ & 2 \\
Women & $30-54$ & 2 \\
TBAs & - & 2 \\
Female opinion leaders & - & 2 \\
Male opinion leaders & - & 2 \\
Males $^{\mathrm{b}}$ & $>30$ & 2 \\
Males $^{\mathrm{c}}$ & $\leq 30$ & 2 \\
\hline
\end{tabular}

\footnotetext{
${ }^{\mathrm{a}}$ One group in Korogocho and another in Viwandani

${ }^{\mathrm{b}}$ Husbands/partners/boyfriends aged above 30 years whose wives/partners had a pregnancy with complications in the last 2 years (2004-2005) preceding the survey

'Husbands/partners/boyfriends aged 30 years and below whose wives/partners had a pregnancy with complications in the last 2 years (2004-2005) preceding the survey
}

attending personnel would already be qualified and would not need to refer the mother to a better-equipped facility. They are however constrained in seeking these services and resort to accessing informal delivery services mostly offered by TBAs or in some cases relatives, friends, or themselves. As a matter of fact, data from this study indicate that TBAs help deliver a majority of the babies born in the two slums. Most of the TBAs interviewed had assisted with the delivery of at least one baby in the month preceding the study. Although widely offering delivery services to these slum communities, most of the TBAs have not had formal training in midwifery, rather, they reported either having inherited their skills or having learned by apprenticeship. A few had taken courses related to delivery offered by the ministry of health.

\section{Barriers to Formal Emergency Obstetric Care Services' Utilization}

Accessing informal delivery services seems to be a common practice in the slums, but when complications set in the birthing mother is forced to seek formal emergency obstetric care. However, when these services are most needed, residents of these two slums face obstacles. The data collected suggest that the major barriers faced include the inability to identify danger signs in time, poor health decision making, unaffordability of health care seeking, poor physical access to formal care services, inadequately equipped health facilities, and poor attitude among health personnel.

Identification of the Danger Signs Reaching a decision to seek medical care when a woman experiences an obstetric complication begins with the ability to correctly recognize the symptoms and signs. Failure to correctly and promptly recognize the symptoms that require a birthing mother to be referred to a formal health facility could act as a barrier or a source of delay. Many participants in the discussions reported that some women in labor take a long time before deciding to seek help. One participant (Women aged 20-29 years) noted that: "I had labor pains for almost a whole week [presumably due to the embarrassment of seeking advice or help to visit a health facility from neighbors]. I got the pain from Sunday to a Thursday. I became very black. I started to rupture on a Monday and kept ignoring until later because I know of some 
neighbors who are just on standby to see how you will behave when you are in labor pain. You have to be strong so that you don't give them something to talk about.". It emerged that a previous history of uncomplicated deliveries limit cause for alarm when complications start, contributing to a delay in deciding to seek help. Additionally, most women in the study areas were reported to seek delivery services from TBAs who live in the community, and TBAs are considered to be committed to the welfare of the community members and have earned their trust. As a result TBAs are considered key opinion leaders on birthing matters, and many women allow them to make decisions on their behalf especially if the husband, who typically considers himself the key decision maker, is unavailable. Many TBAs will promptly refer a case with complications, but in few reported cases the TBA insisted on handling the case, hoping that all would end well. As captured in the words of one TBA: "Some TBAs are also greedy. They keep the woman long past delivery time...they are supposed to refer such a case to hospital..." Also, although not widely reported, attributing curses to complications, also hinder prompt care seeking.

Poor Health Decision Making Correctly recognizing symptoms of an obstetric complication does not automatically translate into positive decision making to seek necessary care. Decision making emerged as a complex issue. Without a supportive spouse, family, or social network, the decision to refer a birthing mother with complications takes a long time to reach. Depending on the structure of decision-making power, the decision could either be made by the woman or any other relative, including the mother-in-law and the husband. Women who rely on their husbands for financial support may not be in a position to make referral decisions without their partners' permission. TBAs reported that they sometimes make referral decisions on behalf of the mother when other family members are absent or when the mother is not in the right mental state to make this decision. In a few of the discussions it emerged that whenever the husband or other family member is uncooperative in decision making, the village chief or headman is sometimes involved. Young women discussants pointed out that their husbands, as providers, are often unwilling to give money for these services, possibly because emphasis is put on allocating meager funds to purchase of food or other essential items.

Unaffordability of Health Care Seeking Respondents in the study recognized poverty as a barrier to the uptake of formal delivery services utilization. They noted that most of slum households are poor and often unable to afford these services, although they want to access formal delivery services. One male respondent noted that most slum residents would want their children to be born in the hospital, however, the high cost of a hospital-based delivery prevents them from realizing this dream. An assessment of facilities accessible to members of these two communities revealed that most of them charge for services related to pregnancy and child birth ${ }^{17}$. All facilities were reported to charge for delivery services, and the price ranged from Kenya Shillings (Ksh) 20 as payment for registration in the government facilities, to Ksh 5,500 in mission hospitals (US \$1= Ksh 80$)^{17}$. This was the amount charged for a normal vaginal delivery, whose average is Ksh 1,700. This fee is much higher for cesarean section, from Ksh 3,000 in a government district hospital to Ksh 30,000 in a private maternity facility. It becomes even more expensive if the birthing mother has to hire a taxi where the 
charges from the slum to some of the formal maternity services in the city of Nairobi can cost as high as Ksh 3,000-4,000. A respondent said that many households find it difficult to pay for these services, and the availability of funds plays a major role in health seeking or lack of it.

As a result, the only option available to most of these women is to seek informal services. Some deliver at home, in an informal facility, or with a TBA. TBAs are less skilled, as mentioned, but often come in handy. Aside from the availability of their services at night, their services are affordable and payment is flexible or free depending on the financial state of the mother. Responding TBAs mentioned that their charges vary, with some mothers being too poor to pay for their services such that the TBA even has to contribute to buying food for the mother if there is none in the house. The highest amount paid was reported to be Ksh 500 (\$6.25). TBAs: "These women do not come to us because they really want to but because of their financial problems. When they think that they will be admitted, given delivery services, then retained at the hospital because they cannot pay the bill, they choose to come to us. It is not their wish really. We do understand and there are some cases, in fact many of them, we do for free [give free services] since they do not have any money."

Lack of money to pay for transport or hire a vehicle to transport a pregnant woman to a health facility was highlighted as a major hindrance to accessing referrals. This, coupled with the cost of care, discouraged the community members from seeking emergency obstetric care. The total amount needed for such an expense is great compared with what an average member of the community earns. Female opinion leaders: "Another reason is money. Delivery services charges as at now is at minimum of Ksh 5,000 (\$62.5) [at a hospital with obstetric services]. Maybe you have also hired a vehicle. You will spend almost Ksh 10,000 (\$125) so she would prefer the nearest and cheapest instead of going all the way to [obstetric specialist hospital]." (Female opinion leaders)

Poor Physical Access to Formal Care Services The unreliable road network within the communities, caused by the haphazard springing up of the slums, emerged as the major infrastructural challenge faced by Korogocho and Viwandani residents. Women with obstetric complications or in labor have to walk or be manually carried to the nearest facility or to public transport out on the main road. It is even more challenging during the rainy season due to the impassable pathways in the slums. It was reported that the situation is worse if complications arise at night, when transport service providers raise their taxi or car-hire charges. Use of matatu (public transport) is affordable, but when not in operation at night, the only means available is use of taxi or car-hire services, which are expensive. Public vehicle operators may sometimes charge even more due to the fear of inconvenience either by having to detour from their route or the woman delivering in the car.

Although reliable and affordable, using public transport presents its own challenges since a pregnant woman still has to walk from the bus terminus to the hospital doorstep and sometimes even stand in the matatu when its full as well as endure traffic jams on the way, causing further delays in getting to a health facility.

With these transportational and financial hindrances, some women are forced to walk all the way to a health facility. This delay causes some to deliver before reaching the facility or to lose their or their babies' lives.

Other factors, which respondents identified as constraining the use of formal emergency obstetric services, include insecurity-especially at night, unfriendly health 
providers, inadequately equipped health facilities, and cumbersome hospital procedures.

Insecurity at Night Insecurity levels in the slums were reported to be very high, a situation that has instilled fear in people of venturing out at night. Loss of property, death, and disabilities arising from attacks by thugs make referrals difficult when a birthing woman experiences complications. Residents fear for their lives. In most cases, they opt instead to use TBA services, putting the lives of the mother and baby at risk from improper procedures. A male discussant (Males over 30 years) narrated his experience: "Like my neighbour last year...she started having labour pains at about 3 p.m. and she did not seem to have prepared anything.... So at about 12 a.m. I was called and found the woman had already given birth and the blood was trickling like water. This problem took me a long time to look for help because at that time Korogocho was very dangerous and you could not even walk at night. We did all we could and we found a certain health worker who finally helped this lady. Then the next day we took her to a major referral hospital in the city because she had lost so much blood."

Unfriendly Health Providers Fear of ill treatment from health workers often discourages women from accessing health facility delivery care. Most discussants cited health professionals especially nurses, midwives, and generally female health providers as having a poor attitude towards pregnant women. With regards to maltreatment during delivery, ownership of the health facility emerged as a major determinant. Participants in the discussions reported that health workers in the formal private facilities-those not owned or run by the government-were more hospitable compared with their counterparts in government facilities who abuse patients, a practice that discouraged women from seeking health facility services when in need. Most women therefore preferred care offered by male birth attendants, opting not to seek care in formal facilities, with the exception especially of Muslim women (because of their belief that it is an abomination to be unlawfully touched by a man). A young female discussant (Women aged 12-19 years) narrated her experience with a government health provider: "On my first visit at [a government clinic] when I was expecting, I was examined but did not wait for further treatment because the doctors [the term 'doctor' is often used to refer to any health care personnel] are very arrogant. I went back home and started going to a private [informal] hospital."

Cumbersome Hospital Procedures/Requirements Hospital procedures or requirements in most hospitals in the country insist on proof of antenatal care attendance and payment of deposit before admittance for care. Pregnant women usually go through the laborious process of registration before eventually queuing to await their turn to be served. It is widely expected by health facilities/providers that pregnant women will go for antenatal care so that in an emergency case, the attending health worker can easily refer to the medical history on the antenatal card. Without an antenatal card, women seeking delivery services are turned away. Further, all women seeking delivery services in health facilities are required to pay a deposit fee before being admitted, and in cases where complications arise an additional amount is charged. Discussants reported that a demand for deposit occurs in all types of health facilities regardless of ownership status. These procedures and requirements discourage women 
from accessing health services. Furthermore, the queues can sometimes be too long such that some women end up delivering while still in the queue. Long queues were blamed on inadequate health professionals at the facilities. Poor women without money to pay for services and those who fail to attend antenatal care stay away from these facilities, even when faced with an obstetric emergency. This quote (Women aged 30-54 years) captures the seriousness given to payment of deposit: "At [a mission hospital], a normal pregnancy requires one to pay a deposit of Ksh 3000 [\$38]. They have put posters all over the place so you do not need to ask anyone. The risky thing is that whether you are their client or not, they cannot take you without the deposit. During admission, you just present the receipt. Be it day or night, you must pay their deposit or else you will just deliver on their doorstep and they will ignore you. I was surprised. With a receipt, the service is very fast...."

It emerged that sometimes these health facilities waive their rules and attend to women with serious emergency complications but detain them after delivery until the bill is settled. Some women have been detained for months, and most worry about being away from their families. This practice has mostly been reported in government health facilities, and although it is not legal, most patients are unaware of this fact and rarely take legal action.

Inadequately Equipped Health Facilities and Poor Accessibility to Referrals The poorly equipped health facilities in the slums are unable to handle emergency cases, and women experiencing complications are referred to facilities away from the slums. Referral of birthing mothers to appropriate facilities for obstetric care is often a complicated process, characterized by communication and transport challenges where the woman and her family often lack the money to pay for transport to the health facility. Even after arriving at a health facility, one may still need to be referred to a better-equipped facility, causing delays in receiving the required services and further deterioration of the patient's condition, thereby lengthening treatment time. The family also always incurs more costs when additional referrals are made. Sometimes the health facilities are equipped with ambulances, but the woman's family is asked to fuel it before she is transferred to a better facility. It was also mentioned that due to frequent stock-outs of drugs in most health facilities, the mother or her family has to buy the prescribed medicines or carry essential delivery materials when going to the facility, failure of which prolongs delay to receive care.

\section{DISCUSSION}

Lay views of the barriers faced by women in accessing emergency obstetric care services in sub-Saharan Africa are understudied. This study investigated the perspectives of poor urban communities in Kenya on the barriers to uptake of formal emergency obstetric care services. It allowed participants to communicate, in their own words, the obstacles they face in accessing emergency obstetric care services, and these qualitative interviews provided a forum for members of Korogocho and Viwandani to freely and exhaustively express their views on this issue. The actionable points/ideas elicited from the interviews gives this study the potential to inform programs and 
policies that will put efforts in place to enhance accessibility of formal emergency obstetric services to women from poor urban settlements in order to reduce incidences of maternal morbidity and mortality. The study reveals that despite slum residents preference of formal health facility delivery, there are obstacles hindering access to formal delivery services. Indeed, recent studies in the slums reveal that a majority of births occur in health facilities most of which are private and located in the informal settlements but are poorly equipped to handle obstetric complications ${ }^{22,25}$. Fotso et al., in their study in the two slums, found that less than a half of women reporting to have delivered in a health facility did this with a skilled attendant ${ }^{22}$. Being informal settlements with temporary structures and lack of proper physical planning by the City Council of Nairobi, the Korogocho, and Viwandani slums have inadequate public health facilities ${ }^{17}$. Most of the better-equipped facilities offering maternity services to these communities are located outside the slums. Being poor, residents are unable to afford transportation and health-care costs to seek care in betterequipped facilities outside the slums ${ }^{23,26}$. Cost is a key factor in determining the choice of place of delivery and also contributes to the first delay, that is, deciding to seek care in a formal health-care facility.

Consequently, a sizeable population in the slums relies on services of TBAs who are seen to have an important role in the community, spanning the pregnancy, delivery, and post-pregnancy periods ${ }^{27}$. They are reported as being trustworthy, and this is important as it builds a sense of confidence in women to always seek information and advice from them. They are often involved in decision making regarding care of a pregnant woman and delivery, and they sometimes help in taking a woman for postnatal care as well as advising on child nutrition, vaccination, and contraception $^{27}$. TBAs often have a flexible mode of payment for their services, making money less of a hindrance in accessing delivery services from them. Their services are also attractive to the populace because they seem to understand the culture of their clientele and are readily available when needed. Although cost emerged as a key determinant in this study, other factors were found to be more important in other studies. Thaddeus and Maine found that people consider the quality of care more than the cost ${ }^{15}$. As reported elsewhere, although the Kenya Ministry of Health recognizes the contribution of TBAs in the health system and has conducted trainings with a few regarding assisting births, there are still some who face obstacles relating with health-care workers ${ }^{27}$.

Ineffective health decision making at the family level is also a major hindrance in accessing formal emergency obstetric care. It emerged that decision making is a complex affair, influenced by individual, societal, access, and health system factors. Timely recognition of the symptoms, availability of finances, autonomy of the birthing mother, physical accessibility of the health facility, and perceived quality of care at the possible referral facilities are necessary considerations in making the decision to seek formal care. As was found in a study in Haiti, decision making can cause delays in receiving the required care ${ }^{28}$. In this study, recognition of the danger signs was very important in referral decision making. And as Cham et al. found in their maternal study in the rural Gambia, underestimation of the severity of the obstetric complication may cause delays in receiving the required care ${ }^{29}$. In Southern Tanzania, perceptions and interpretations by the rural community of pregnancy-related risks determine whether they self-treat or seek health facility care on time ${ }^{30}$. In our study, it emerged that women who have had previous safe deliveries are not immediately thought to need or require emergency care when obstetric complications 
arise. Some TBAs who assumed they would be able to handle obstetric complications were also blamed for delays in referrals.

Although distance and cost have been established as the major barriers to health care $^{15,18,31-33}$, in this study, distance did not emerge as a major issue in accessing emergency obstetric care because of the readily available health facilities close to the slums. Unlike rural populations, residents of the Korogocho and Viwandani slums do not face long distances to health facilities, due to their location in the city of Nairobi. Availability of adequate transport facilities when needed is, however, also an important determinant of access of the needed care. Using data from the Demographic and Health Survey to understand the barriers to utilization of maternal health care in rural Mali for instance, Gage found transport to be important in delivery outcomes. Elsewhere, lack of transport facilities makes it difficult for women who go into labor or develop obstetric complications at night to get to a health facility. A study in western Kenya found that of the $83 \%$ of women interviewed who delivered outside a health facility, $49 \%$ cited lack of transport to access a facility, especially at night, while $28 \%$ cited expense as a major barrier ${ }^{32}$. Another study seeking to understand the barriers to treatment of obstetric emergencies among rural communities also found distance from health facilities and transportation challenges as key in accessing services ${ }^{33}$. Residents of Nairobi slums, however, do not seem to face the same barriers in transport as rural communities. Being in an urban area, transport facilities offered by minibuses are adequate especially during the day, but they pick and drop residents at the periphery of the slums because of the poor road network there. The unique transport challenge faced by this group therefore seems to be the inaccessibility of the structures due to the poor road network forcing a birthing mother to walk to the bus stop even while her condition worsens. The transport situation therefore seems to be different between the urban and rural populations. While difficulties in obtaining transport in rural Gambia contributed to delayed care seeking, what hindered formal care seeking in this study was the cost of transport involved, especially at night when public transport is not in operation and one has to hire a taxi. Insecurity at night due to fear of attacks by thugs was also found to be a key determinant of whether birthing women in these slums seek obstetric care.

Some of the obstacles existing at the health facility level in this study have been reported elsewhere ${ }^{18,29}$. Inadequately equipped health facilities have been reported to either cause delays in receiving appropriate care or necessitating additional referrals to better-equipped facilities to be made. Inadequacy of health personnel forces the patients to queue for long hours, while unfriendly health providers and laborious health facility procedures, such as demand for deposit or antenatal card, discourage care seeking. A recent study in Gambia investigating women users' perspectives of the availability and quality of emergency obstetric care services at the main referral hospital in the capital city found various health system inadequacies including lack of blood for transfusion, shortage of essential medicines, improper reception at the referral hospital, difficulty in obtaining cesarean section, and perceived poor quality of care received ${ }^{29}$. The Gambia study, although using a qualitative approach in data collection, only focused on the women users' perspectives and only studied the barriers at the facility level; hence, the study does not present the contribution of the other individual, social, and community factors on health services utilization. Similar to a study in Nigeria, our study found unfriendliness of staff and the promptness of care to be hindrances when seeking obstetric emergency care ${ }^{18}$. By collecting the views of the women users, their partners, TBAs, and opinion leaders, 
this study expands the knowledge of barriers to use of formal health services at different levels including household/family, community, and health facility levels.

The practice of detaining insolvent women patients at health facilities due to the inability to clear their medical bills could deter more of them in need of formal health care from seeking it. Detention of patients is a common practice in health facilities in developing countries that lack a proper medical scheme to cushion the poor, most of whom are unable to pay the hospital bills incurred. This is because of the cost-sharing mechanism where patients are expected to pay for part of the health-care costs in government facilities or pay for the full cost in private or faithbased facilities. Patients under detention usually face various conditions including overcrowding, insufficient food and water, and withholding of further medical treatment ${ }^{34}$.

\section{CONCLUSION}

The hindrances identified in this study can be summed up as contributing to the three delays in accessing emergency obstetric care services. Borrowing from the study by Thaddeus and Maine on the Three-Delays Model $^{15}$, this study recommends improving availability, accessibility, and quality of emergency obstetric services to these slums. The authors note that the factors contributing to the delays in seeking these services rarely work in isolation and advise planners to target all specific barriers as highlighted in the study. In line with this recommendation, planners also need to consider the recommendations of the 2009 Women Deliver conference in London to increase the number of skilled birth attendants in order to enable women in a resource-poor urban setting like the Nairobi slums to access modern maternal health services so that they can have safe births $^{35}$. Due to the overwhelming preference of TBA services despite their inadequacies, this study supports strategies involving TBAs in maternal health care delivery. Health facilities can work in collaboration with TBAs because they have the potential to ensure effective referrals. In turn, facilities can offer supervision or support to TBAs to ensure that their work in the community safeguards the health of women and their children. The Kenya Ministry of Health can also develop strategies for offering TBA trainings that can enable them to handle minor complications and determine those to be referred on time in order to limit the three delays. There is also a need to strengthen the existing support systems in order to ensure that facilities in the community are improved to offer the minimum-standard delivery care and ensure that timely referrals are made when necessary. Training for health workers in formal health facilities regarding client public relations is also important if women are to have faith in the formal health care system. There is also need to strengthen links between communities and facilities by the health facility boards and committees in order to encourage community members to access formal health services, which can greatly reduce death and disability arising from obstetric complications. Finally, there is a need for researchers to make use of qualitative studies in order to understand the dynamics of obstacles to obstetric health care access at the household and community levels.

Of ultimate importance is the possibility of implementing the fee-exemption policy for pregnant women so that they are not detained at health facilities due to inability to pay for delivery services, a practice that could discourage them from seeking formal care. 


\section{ACKNOWLEDGMENTS}

The authors thank Dr. Chimaraoke Izugbara and Ms. Elizabeth Kimani-Murage both from the African Population and Health Research Center (APHRC) for reviewing earlier versions of the manuscript. They acknowledge the financial support from the World Bank Netherlands Partnership Program that enabled the collection of the data used in this study (grant no. 713 6587) and the design of the research questions addressed in this paper (grant no. 304 406-29). Their time was funded by the Wellcome Trust (grant no. GR 078 530M).

Competing interests. The authors declare that they do not have any competing interests.

Authors' contributions. H.E. did the analysis of data and the literature review, wrote the first draft of the paper, and reviewed the final draft. S.M. participated in the planning of the study and was involved in the conception of the study, literature review, analysis, and review of the paper. J.C.F. participated in the planning of the study and data collection and was involved in the conception of the paper, literature review, and the review of the manuscript. All authors reviewed the final manuscript.

\section{REFERENCES}

1. Ronsmans C, Graham WJ. Maternal mortality: who, when, where and why. Lancet. 2006; 368: 1189-1200.

2. Starrs A. Safe motherhood initiative: 20 years and counting. Lancet. 2006; 368: 1130-1132.

3. United Nations. The millennium development goals report 2006. New York: United Nations; 2006.

4. Paxton A, Maine D, Freedman L, Fry D, Lobis S. The evidence for emergency obstetric care. Int J Gynecol Obstet. 2005; 88: 181-193.

5. World Health Organization. Proportion of births attended by a skilled health worker, 2008 updates. Geneva: World Health Organization; 2008.

6. Campbell OMR, Graham WJ. Strategies for reducing maternal mortality: getting on with what works. Lancet. 2006; 368: 1284-1299.

7. United Nations. The millennium development goals report. New York: United Nations; 2009.

8. WHO/UNICEF/UNFPA. Maternal mortality in 2000: estimates developed by WHO, UNICEF and UNFPA. Geneva: World Health Organization; 2004.

9. CentralBureauof Statistics(CBS)[Kenya],MinistryofHealth(MoH)[Kenya],ORCMacro.Kenya Demographic and Health Survey 2003. Calverton, MD: CBS, MoH, and ORC Macro; 2004.

10. Ziraba AK, Madise N, Mills S, Kyobutungi C, Ezeh A. Maternal mortality in the informal settlements of Nairobi city: what do we know? Reprod Health. 2009; 6: 6.

11. APHRC (African Population and Health Research Center). Population and health dynamics in Nairobi's informal settlements. Nairobi: African Population and Health Research Center; 2002.

12. Central Bureau of Statistics. Welfare Monitoring Survey III. Government Priorities ed. Nairobi: Ministry of Planning and National Development; 2000.

13. Cohen B. Urban growth in developing countries: a review of current trends and a caution regarding existing forecast. World Dev. 2004; 32: 23-51.

14. Brockerhoff MP, Brennan E. The poverty of cities in developing countries. Popul Dev Rev. 1998; 24: 75-114.

15. Thaddeus S, Maine D. Too far to walk: maternal mortality in context. Soc Sci Med. 1994; 38: 1091-1110.

16. Magadi M, Diamond I, Madise N. Analysis of factors associated with maternal mortality in Kenyan hospitals. J Biosoc Sci. 2001; 33: 375-389. 
17. African Population and Health Research Center (APHRC), The World Bank. Averting preventable maternal mortality: delays and barriers to the utilization of emergency obstetric care in Nairobi's informal settlements. Nairobi: APHRC and The World Bank; 2006.

18. Onah HE, Ikeako LC, Iloabachie GC. Factors associated with the use of maternity services in Enugu, southeastern Nigeria. Soc Sci Med. 2006; 63: 1870-1878.

19. Cham M, Sundby J, Vangen S. Maternal mortality in the rural Gambia, a qualitative study on access to emergency obstetric care. Reprod Health. 2005; 2: 3.

20. Gage AJ. Barriers to the utilization of maternal health care in rural Mali. Soc Sci Med. 2007; 65: 1666-1682.

21. Ziraba AK, Mills S, Madise N, Saliku T, Fotso JC. The state of emergency obstetric care services in Nairobi informal settlements and environs: results from a maternity health facility survey. BMC Health Serv Res. 2009; 9: 46.

22. Fotso JC, Ezeh A, Madise N, Ziraba A, Ogollah R. What does access to maternal health care mean among the urban poor? Factors associated with the use of appropriate maternal health services in the slum settlements of Nairobi, Kenya. Matern Child Health. 2008; 13: 130-137.

23. Magadi MA, Zulu EM, Brockerhoff M. The inequality of maternal health care in urban sub-Saharan Africa in the 1990s. Pop Stud. 2003; 57: 347-366.

24. Creswell JW. Qualitative inquiry and research design: choosing among five traditions. Thousand Oaks: Sage; 1998.

25. Bazant ES, Koenig MA, Fotso JC, Mills S. Women's use of private and government health facilities for childbirth in Nairobi's informal settlements. Stud Fam Plann. 2009; 40: 3950.

26. Fotso JC, Ezeh A, Oronje R. Provision and use of maternal health services among urban poor in Kenya: what do we know and what can we do? J Urban Health. 2008; 85: 428-442.

27. Izugbara C, Ezeh A, Fotso JC. The persistence and challenges of homebirths: perspectives of traditional birth attendants in urban Kenya. Health Policy Plann. 2009; 24: 36-45.

28. Barnes-Josiah D, Myntti C, Augustin A. The "three delays" as a framework for examining maternal mortality in Haiti. Soc Sci Med. 1998; 46: 981-993.

29. Cham M, Sundby J, Vangen S. Availability and quality of emergency obstetric care in Gambia's main referral hospital: women-users' testimonies. Reprod Health. 2009; 6: 5.

30. Kowalewski M, Jahn A, Kimatta SS. Why do at-risk mothers fail to reach referral level? Barriers beyond distance and cost. Afri J Reprod Health. 2000; 4: 100-109.

31. Amooti-Kaguna B, Nuwaha F. Factors influencing choice of delivery sites in Rakai district of Uganda. Soc Sci Med. 2000; 50: 203-213.

32. Van Eijk AM, Bles HM, Odhiambo F, et al. Use of antenatal services and delivery care among women in rural western Kenya: a community based survey. Reprod Health. 2006; 3: 2.

33. The Prevention of Maternal Mortality Network. Barriers to treatment of obstetric emergencies in rural communities of West Africa. Stud Fam Plann. 1992; 23: 279-291.

34. Kippenberg J, Sahokwasama JB, Amon JJ. Detention of insolvent patients in Burundian hospitals. Health Policy Plann. 2007; 23: 14-23.

35. Family Care International, Recommendations of the Women Deliver Conference, London 2007. Women Deliver Web site. http://www.womendeliver.org/resources/pdf/Focuson5. pdf. Accessed September 3, 2009. 